\title{
TFAP2B, AP-1 and $J A Z F 1$ Expression in Tissues of Papillary Thyroid Carcinoma Patients; Clinical, Pathological and Prognostic Values
}

\author{
Abdulwahab A Abuderman*, Ola A Harb², Loay M Gertallah³, Nahlah Makki \\ Almansour ${ }^{4}$
}

\begin{abstract}
Objective: Transcription factor activating protein 2 B (TFAP2 B) is a transcription factor that regulates many steps of embryogenesis, cell growth, apoptosis and recently oncogenesis and cancer progression. AP-1 is a transcription factor that is a downstream molecule of the MAPK signaling pathway. Juxtaposed with zinc finger gene $1(J A Z F 1)$ is a recently detected transforming growth factor which has a role in carcinogenesis. Hence the present study aimed to assess those markers expression in tissues from patients with such cancer correlation their expression with clinic-pathological findings of the tumor and prognostic and follow-up findings of patients. Methods: We have collected tissue samples from papillary thyroid cancer patients and adjacent non-neoplastic tissues from 80 patients. We assessed the expression of TFAP2B, AP-1 and JAZF1 using immunohistochemistry. Results: Expression of TFAP2B was positively associated with lymph nodes metastases $(\mathrm{p}=0.003)$, distant metastases $(\mathrm{p}=0.002)$, recurrence of the tumor $(\mathrm{p}=0.002)$, unfavourable disease-free survival rate $(\mathrm{p}=0.003) . A P-1$ expression is positively associated with advanced stage $(\mathrm{p}=0.002)$, presence of extra-thyroid invasion ( $\mathrm{p}=0.005)$, recurrence of the tumor $(\mathrm{p}=0.005)$, unfavorable disease-free survival rate $(\mathrm{p}=0.01)$. $J A Z F 1$ expression is negatively associated with huge tumor size $(0.023)$, vascular invasion $(\mathrm{p}=0.007)$ and unfavorable overall survival rate $(\mathrm{p}=.030)$. Conclusion: High expression levels of TFAP $2 B$ and $A P-1$ and low expression levels of JAZF1 were associated with unfavourable pathological, prognostic parameters and dismal patient's outcome.
\end{abstract}

Keywords: Papillary thyroid cancer- TFAP2B- AP-1- JAZF1- prognosis- immunohistochemistry

Asian Pac J Cancer Prev, 21 (8), 2415-2421

\section{Introduction}

Thyroid cancer is considered the most prevalent endocrine system cancers that continued rising worldwide in the past decades (Xiao et al., 2019). Papillary carcinoma of the thyroid formed about $74-80 \%$ of all thyroid malignancies (Huang et al., 2019). Papillary thyroid cancer has a favourable patients outcome and better patients survival, but it was found that some patients might have more aggressive phenotypes with lack of response to the currently used therapies with high patients morbidity and mortality (Fu et al., 2019). Therefore, identifying new mechanisms of cancer progression and novel molecular biomarkers might help in the identification of patients having a higher liability of papillary carcinoma of the thyroid recurrence and progression. Transcription factor activating protein $2 \mathrm{~B}$ (TFAP2 B) is a transcription factor that regulates many steps of embryogenesis, cell growth, apoptosis and recently oncogenesis and cancer progression
(Xu et al., 2017, Fu et al., 2019). AP-1 is a transcription factor that is a downstream molecule of the MAPK signaling pathway, which is incriminated in controlling many cellular processes as cell growth, apoptosis and proliferation (Li et al., 2016). Disturbances in the expression of $A P-1$ were linked to tumor transformation, progression, invasion, metastases and angiogenesis (Tewari et al., 2018). Juxtaposed with zinc finger gene 1 $(J A Z F 1)$ is a recently detected transforming growth factor which has a role in carcinogenesis (Nakajima et al., 2004). As no previous studies have assessed the roles of TFAP2B, AP-1 and JAZF1 expression in patients with papillary carcinoma of the thyroid, we aimed to assess TFAP2B, AP-1 and JAZF1 expression in tissues from patients with such cancer correlation their expression with clinic-pathological findings of the tumor and prognostic and follow-up findings of patients.

${ }^{1}$ Department of Basic Medical Sciences, College of Medicine, Prince Sattam Bin Abdulaziz University, Al-Kharj, Saudi Arabia.. ${ }^{2}$ Department of Pathology, Faculty of Medicine, Zagazig University, Zagazig, Egypt. ${ }^{3}$ Department of General Surgery, Faculty of Medicine, Zagazig University, Zagazig, Egypt. ${ }^{4}$ Department of Biology, College of Science, University of Hafr Al Batin, Hafr Al Batin, Saudi Arabia.*For Correspondence: a.abuderman@psau.edu.sa 


\section{Materials and Methods}

We had collected tissue samples from papillary thyroid cancer patients and adjacent non-neoplastic tissues from 80 patients who were operated in the University's Teaching Hospitals and other Private Hospitals.

\section{Inclusion criteria}

Patients with a confirmed diagnosis of papillary thyroid carcinoma, Patients with completed clinical data, Patients who accepted to be included in the study, patients without a history of pre-operative chemotherapy or radiotherapy treatment

\section{Exclusion criteria}

Patients with incomplete data, patients who have another cancer, patients received any pre-operative treatment; all included patients provided written informed consent to be included in the study. An approval for performing the study was taken from the local ethical committee of the institutional review board of Zagazig University, Faculty of Medicine, Egypt.”

\section{Immunohistochemistry technique}

The tissues which were collected from the surgical samples have been embedded in a paraffin block, cut at a thickness of $3-\mu \mathrm{m}$ for performing immunohistochemistry by incubation with primary polyclonal anti; TFAP2B, AP-1 and JAZF1 antibodies at dilution 1:100 (Abcam, Cambridge, UK).

\section{Assessment of TFAP2B, AP-1 and JAZF1 expression in stained tissues}

We have assessed both staining extent and intensity; staining extent was estimated on scores from 1-4 (0, negative; 1 less than $10 \% ; 2$ from 10 to $50 \% ; 3$ from 51 to $80 \%$; 4 more than $80 \%$ ). The staining intensity was estimated on scores from 1-3 (0, negative staining; 1 represented weak staining; 2, represented moderate staining; 3represented strong staining. The final score of TFAP2B, AP-1 and JAZF1 was reached by multiplying scores of extent and intensity, 2 was the cut point, and $\geq 5$ was positive expression while below 5 is negative.

\section{Statistical analysis}

For data analysis we have used software statistical analysis program SSSP 24.0 (Chicago, USA) was used for data analysis, using; Chi-square test for analysis of differences in protein expression of TFAP $2 B, A P-1$ and $J A Z F 1$ in collected tissues. Kaplan Meier plots were used to assess patients' survival. $\mathrm{P}<0.05$ was considered a statistically significant value.

\section{Results}

We included 80 patients (22 males, 58 females), with their age, ranging from 16 to 70 years. There were 48 patients have cervical lymph node spread, and 26 patients have an extra-thyroid invasion. We included samples from 80 papillary thyroid cancers; 72 of them were diagnosed as having conventional papillary thyroid carcinoma, and 8 of them were diagnosed as having a follicular variant of papillary thyroid carcinoma and 80 samples from adjacent non-neoplastic thyroid tissue of the same patients (Table 1).

\section{TFAP2B expression in thyroid tissue Immunohistochemistry results}

Expression of TFAP2B was elevated in samples from papillary thyroid carcinoma more than samples from adjacent non-neoplastic thyroid tissue of the same patients $(\mathrm{p}=0.002)$. TFAP $2 B$ expression in papillary thyroid cancer tissues is positively associated with advanced stage, lymph nodes metastases $(p=0.003)$, huge tumor size

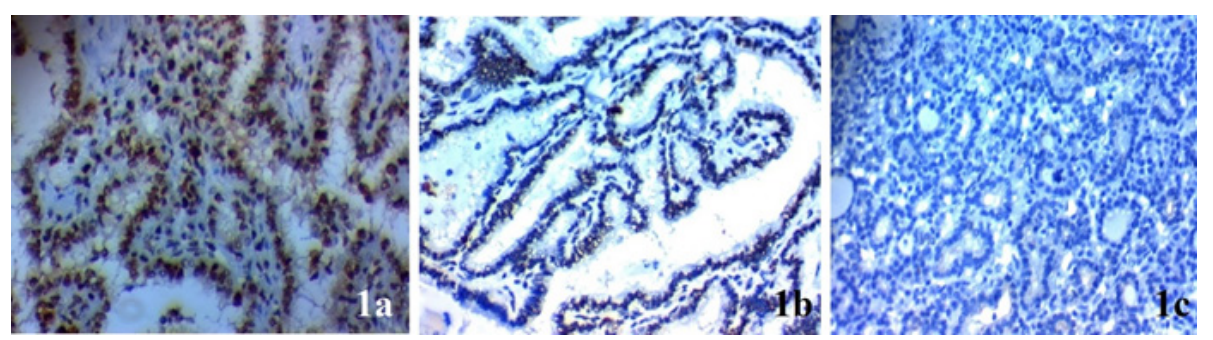

Figure 1.TFAP2B Expression in Papillary Thyroid Carcinoma (PTC). (A) High cytoplasmic expression in poorly differentiated PTC of high stage $\times 400$, (B) low cytoplasmic expression in well differentiated PTC of low stage $\times 400$, (C) negative cy
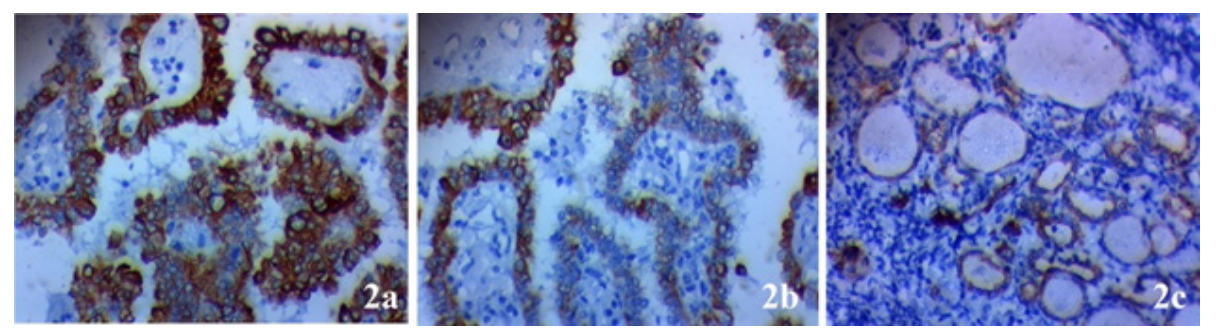

Figure 2. AP-1 Expression in Papillary Thyroid Carcinoma (PTC). (A) High nuclear expression in poorly differentiated PTC of high stage $\times 400$, (B) low nuclear expression in well differentiated PTC of low stage $\times 400$, (C) negative nuclear exp 
DOI: 10.31557/APJCP.2020.21.8.2415

TFAP2B, AP-1 and JAZF1 Expression in Tissues of Papillary Thyroid Carcinoma Patients

Table 1. Association of Clinicopathological Features with TFAP2B, AP-1 and JAZF1 Expression in Included Papillary Thyroid Carcinoma Patients

\begin{tabular}{|c|c|c|c|c|c|c|c|c|c|c|c|c|c|c|c|c|}
\hline & & \multicolumn{4}{|c|}{ TFAP2B } & $\mathrm{p}$ & \multicolumn{4}{|c|}{ AP-1 } & $\mathrm{p}$ & \multicolumn{4}{|c|}{ JAZF1 } & \multirow[t]{3}{*}{$\mathrm{p}$} \\
\hline & & \multirow{2}{*}{\multicolumn{2}{|c|}{$\begin{array}{c}\text { Low } \\
\mathrm{N}=30\end{array}$}} & \multirow{2}{*}{\multicolumn{2}{|c|}{$\begin{array}{l}\text { High } \\
\mathrm{N}=50\end{array}$}} & & \multirow{2}{*}{\multicolumn{2}{|c|}{$\begin{array}{l}\text { Low } \\
\mathrm{N}=42\end{array}$}} & \multirow{2}{*}{\multicolumn{2}{|c|}{$\begin{array}{l}\text { High } \\
\mathrm{N}=38\end{array}$}} & & \multirow{2}{*}{\multicolumn{2}{|c|}{$\begin{array}{l}\text { Low } \\
\mathrm{N}=50\end{array}$}} & \multirow{2}{*}{\multicolumn{2}{|c|}{$\begin{array}{l}\text { High } \\
\mathrm{N}=30\end{array}$}} & \\
\hline & & & & & & & & & & & & & & & & \\
\hline \multirow[t]{2}{*}{ Age.Group } & $<40 y$ & 12 & $40.00 \%$ & 28 & $56.00 \%$ & 0.337 & 14 & $33.30 \%$ & 26 & $68.40 \%$ & 0.033 & 30 & $60.00 \%$ & 10 & $33.30 \%$ & 0.002 \\
\hline & $\Rightarrow>40 y$ & 18 & $60.00 \%$ & 22 & $44.00 \%$ & & 28 & $66.70 \%$ & 12 & $31.60 \%$ & & 20 & $40.00 \%$ & 20 & $66.70 \%$ & \\
\hline \multirow[t]{2}{*}{ Sex } & M & 10 & $33.30 \%$ & 12 & $24.00 \%$ & 0.422 & 12 & $28.60 \%$ & 10 & $26.30 \%$ & 0.233 & 14 & $28.00 \%$ & 8 & $26.70 \%$ & 0.827 \\
\hline & F & 20 & $66.70 \%$ & 38 & $76.00 \%$ & & 30 & $71.40 \%$ & 28 & $73.70 \%$ & & 36 & $72.00 \%$ & 22 & $73.30 \%$ & \\
\hline \multirow{2}{*}{$\begin{array}{l}\text { Histo- } \\
\text { pathological } \\
\text { subtype }\end{array}$} & PTC conv & 28 & $93.30 \%$ & 44 & $88.00 \%$ & 0.346 & 40 & $95.20 \%$ & 32 & $84.20 \%$ & 0.546 & 44 & $88.00 \%$ & 28 & $93.30 \%$ & 0.586 \\
\hline & PTC FV & 2 & $6.70 \%$ & 6 & $12.00 \%$ & & 2 & $4.80 \%$ & 6 & $15.80 \%$ & & 6 & $12.00 \%$ & 2 & $6.70 \%$ & \\
\hline \multirow[t]{4}{*}{ Stage } & I & 12 & $40.00 \%$ & 2 & $4.00 \%$ & 0.003 & 14 & $33.30 \%$ & 0 & $0.00 \%$ & 0.002 & 6 & $12.00 \%$ & 8 & $26.70 \%$ & 0.034 \\
\hline & II & 14 & $46.70 \%$ & 8 & $16.00 \%$ & & 18 & $42.90 \%$ & 4 & $10.50 \%$ & & 8 & $16.00 \%$ & 14 & $46.70 \%$ & \\
\hline & III & 2 & $6.70 \%$ & 26 & $52.00 \%$ & & 4 & $9.50 \%$ & 24 & $63.20 \%$ & & 24 & $48.00 \%$ & 4 & $13.30 \%$ & \\
\hline & IV & 2 & $6.70 \%$ & 14 & $28.00 \%$ & & 6 & $14.30 \%$ & 10 & $26.30 \%$ & & 12 & $24.00 \%$ & 4 & $13.30 \%$ & \\
\hline \multirow[t]{2}{*}{ Tumor size } & $<4 \mathrm{~cm}$ & 26 & $86.70 \%$ & 24 & $48.00 \%$ & 0.022 & 34 & $81.00 \%$ & 16 & $42.10 \%$ & 0.034 & 24 & $48.00 \%$ & 26 & $86.70 \%$ & 0.023 \\
\hline & $=>4 \mathrm{~cm}$ & 4 & $13.30 \%$ & 26 & $52.00 \%$ & & 8 & $19.00 \%$ & 22 & $57.90 \%$ & & 26 & $52.00 \%$ & 4 & $13.30 \%$ & \\
\hline \multirow{4}{*}{$\begin{array}{l}\text { Surgery } \\
\text { (thyroidectomy) }\end{array}$} & Total & 12 & $40.00 \%$ & 28 & $56.00 \%$ & 0.03 & 20 & $47.60 \%$ & 20 & $52.60 \%$ & 0.031 & 28 & $56.00 \%$ & 12 & $40.00 \%$ & 0.094 \\
\hline & Subtotal & 10 & $33.30 \%$ & 4 & $8.00 \%$ & & 12 & $28.60 \%$ & 2 & $5.30 \%$ & & 4 & $8.00 \%$ & 10 & $33.30 \%$ & \\
\hline & $\begin{array}{l}\text { Total } \\
\text { +BND }\end{array}$ & 8 & $26.70 \%$ & 16 & $32.00 \%$ & & 8 & $19.00 \%$ & 16 & $42.10 \%$ & & 18 & $36.00 \%$ & 6 & $20.00 \%$ & \\
\hline & lobectomy & 0 & $0.00 \%$ & 2 & $4.00 \%$ & & 2 & $4.80 \%$ & 0 & $0.00 \%$ & & 0 & $0.00 \%$ & 2 & $6.70 \%$ & \\
\hline \multicolumn{2}{|l|}{ Multifocality } & 6 & $13.30 \%$ & 39 & $60.00 \%$ & 0.002 & 8 & $19.00 \%$ & 26 & $68.40 \%$ & 0.003 & 28 & $56.00 \%$ & 6 & $20.00 \%$ & 0.036 \\
\hline \multicolumn{2}{|l|}{ LN involvement } & 8 & $26.70 \%$ & 40 & $80.00 \%$ & 0.003 & 12 & $28.60 \%$ & 36 & $94.70 \%$ & 0.002 & 38 & $76.00 \%$ & 10 & $33.30 \%$ & 0.005 \\
\hline \multicolumn{2}{|l|}{ Vascular invasion } & 4 & $6.70 \%$ & 18 & $36.00 \%$ & 0.028 & 6 & $14.30 \%$ & 14 & $36.80 \%$ & 0.02 & 16 & $32.00 \%$ & 4 & $13.30 \%$ & 0.007 \\
\hline \multicolumn{2}{|l|}{ Capsular invasion } & 4 & $6.70 \%$ & 28 & $56.00 \%$ & 0.004 & 8 & $19.00 \%$ & 22 & $57.90 \%$ & 0.022 & 24 & $48.00 \%$ & 6 & $20.00 \%$ & 0.037 \\
\hline \multicolumn{2}{|c|}{ Extrathyroid extension } & 4 & $6.70 \%$ & 24 & $48.00 \%$ & 0.005 & 6 & $14.30 \%$ & 20 & $52.60 \%$ & 0.032 & 22 & $44.00 \%$ & 4 & $13.30 \%$ & 0.045 \\
\hline \multicolumn{2}{|l|}{ Distant metastasis } & 4 & $6.70 \%$ & 14 & $28.00 \%$ & 0.002 & 6 & $14.30 \%$ & 10 & $26.30 \%$ & 0.042 & 12 & $24.00 \%$ & 4 & $13.30 \%$ & 0.414 \\
\hline
\end{tabular}

$(\mathrm{p}=0.022)$, presence of extra-thyroid invasion $(\mathrm{p}=0.005)$, multifocality of the tumor $(\mathrm{p}=0.002)$, vascular invasion $(\mathrm{p}=0.028)$, capsular invasion $(\mathrm{p}=0.004)$ and distant metastases $(p=0.002)$ (Table 1 and Figure 1-3).
Patients' outcome and survival results

$T F A P 2 B$ expression in papillary thyroid cancer tissues is positively associated with a high incidence of disease progression, recurrence of the tumor $(p=0.002)$, unfavourable disease-free survival rate $(p=0.003)$ (Tables 2-4 and Figures 4-5).

Table 2. The Outcome of Patients in Relation to TFAP2B, AP-1 and JAZF1 Expression in Included Papillary Thyroid Carcinoma Patients

\begin{tabular}{|c|c|c|c|c|c|c|c|c|c|c|c|c|c|c|c|}
\hline & \multicolumn{4}{|c|}{ TFAP2B } & $\mathrm{p}$ & \multicolumn{4}{|c|}{ AP-1 } & $\mathrm{p}$ & \multicolumn{4}{|c|}{ JAZF1 } & \multirow[t]{3}{*}{$\mathrm{p}$} \\
\hline & \multirow{2}{*}{\multicolumn{2}{|c|}{$\begin{array}{l}\text { Low } \\
\mathrm{N}=30\end{array}$}} & \multirow{2}{*}{\multicolumn{2}{|c|}{$\begin{array}{l}\text { High } \\
\mathrm{N}=50\end{array}$}} & & \multirow{2}{*}{\multicolumn{2}{|c|}{$\begin{array}{l}\text { Low } \\
\mathrm{N}=42\end{array}$}} & \multirow{2}{*}{\multicolumn{2}{|c|}{$\begin{array}{l}\text { High } \\
\mathrm{N}=38\end{array}$}} & & \multirow{2}{*}{\multicolumn{2}{|c|}{$\begin{array}{l}\text { Low } \\
\mathrm{N}=50\end{array}$}} & \multirow{2}{*}{\multicolumn{2}{|c|}{$\begin{array}{l}\text { High } \\
\mathrm{N}=30\end{array}$}} & \\
\hline & & & & & & & & & & & & & & & \\
\hline \multicolumn{16}{|l|}{ Response } \\
\hline $\mathrm{PD}$ & 0 & $0.00 \%$ & 0 & $0.00 \%$ & 0.03 & 0 & $0.00 \%$ & 0 & $0.00 \%$ & 0.004 & 0 & $0.00 \%$ & 0 & $0.00 \%$ & 0.0872 \\
\hline SD & 0 & $0.00 \%$ & 2 & $4.00 \%$ & & 0 & $0.00 \%$ & 2 & $5.30 \%$ & & 2 & $4.00 \%$ & 0 & $0.00 \%$ & \\
\hline PR & 0 & $0.00 \%$ & 4 & $8.00 \%$ & & 2 & $4.80 \%$ & 2 & $5.30 \%$ & & 2 & $4.00 \%$ & 2 & $6.70 \%$ & \\
\hline $\mathrm{CR}$ & 30 & $100.00 \%$ & 44 & $88.00 \%$ & & 40 & $95.20 \%$ & 34 & $89.50 \%$ & & 46 & $92.00 \%$ & 28 & $93.30 \%$ & \\
\hline \multicolumn{16}{|c|}{ Overall Response } \\
\hline NoCR & 0 & $0.00 \%$ & 6 & $12.00 \%$ & 0.049 & 2 & $4.80 \%$ & 4 & $10.50 \%$ & 0.479 & 4 & $8.00 \%$ & 2 & $6.70 \%$ & 0.347 \\
\hline $\mathrm{CR}$ & 30 & $100.00 \%$ & 44 & $88.00 \%$ & & 40 & $95.20 \%$ & 34 & $89.50 \%$ & & 46 & $92.00 \%$ & 28 & $93.30 \%$ & \\
\hline \multicolumn{16}{|l|}{ Recurrence } \\
\hline No & 26 & $86.70 \%$ & 14 & $28.00 \%$ & 0.002 & 30 & $71.40 \%$ & 10 & $26.30 \%$ & 0.005 & 18 & $36.00 \%$ & 22 & $73.30 \%$ & 0.031 \\
\hline Yes & 4 & $13.30 \%$ & 30 & $60.00 \%$ & & 10 & $23.80 \%$ & 24 & $63.20 \%$ & & 28 & $56.00 \%$ & 6 & $20.00 \%$ & \\
\hline \multicolumn{16}{|l|}{ Outcome } \\
\hline Censored & 26 & $86.70 \%$ & 40 & $80.00 \%$ & 0.041 & 38 & $90.50 \%$ & 28 & $73.70 \%$ & 0.053 & 38 & $76.00 \%$ & 28 & $93.30 \%$ & 0.162 \\
\hline Died & 4 & $13.30 \%$ & 10 & $20.00 \%$ & & 4 & $9.50 \%$ & 10 & $26.30 \%$ & & 12 & $24.00 \%$ & 2 & $6.70 \%$ & \\
\hline
\end{tabular}


Table 3. Survival Analysis in Whole PTC Group and in Relation to TFAP2B, AP-1 and JAZF1 Expression in Included Papillary Thyroid Carcinoma Patients

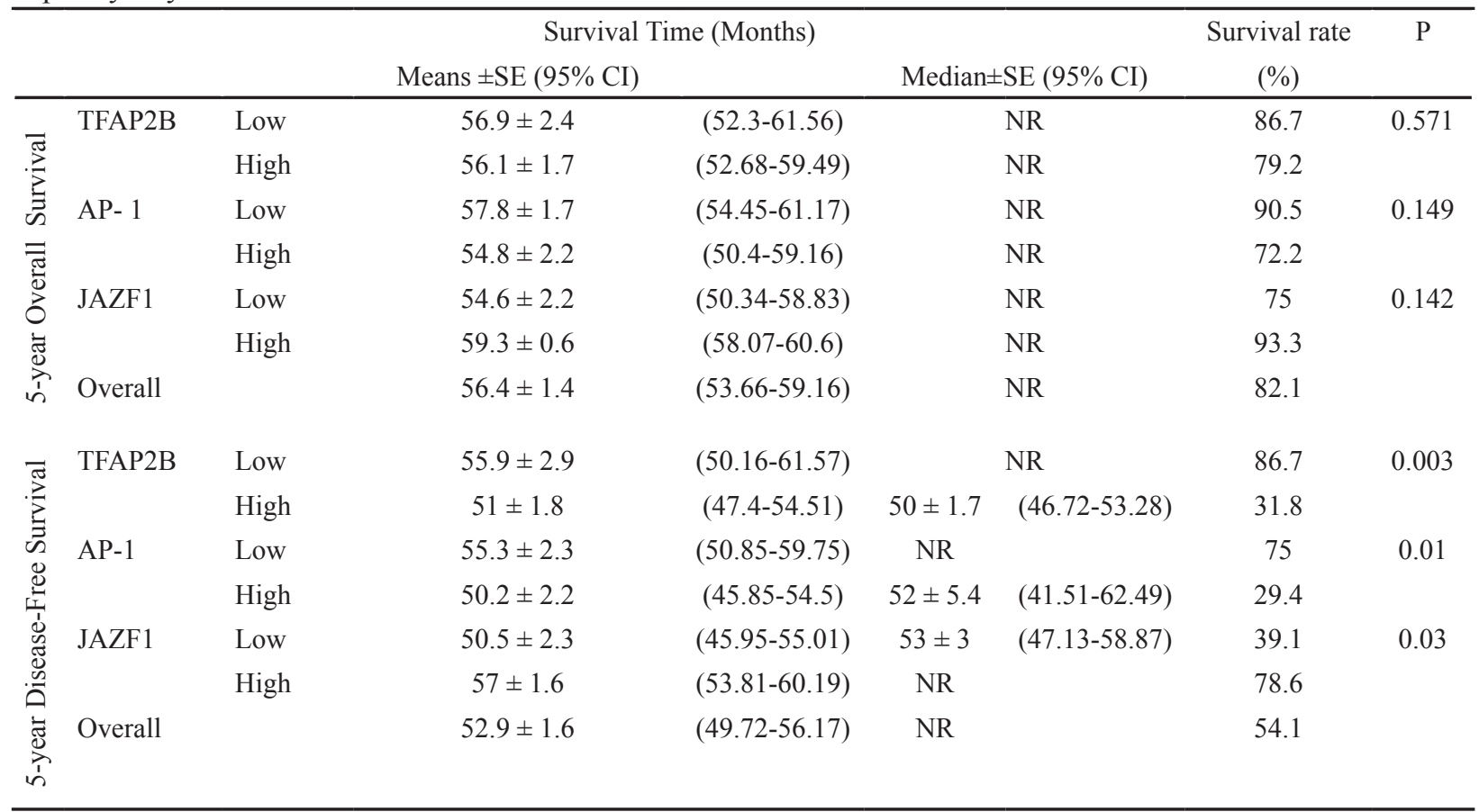

NR, not reached

\section{AP-1 expression in thyroid tissue}

\section{Immunohistochemistry results}

Expression of AP-1was elevated in samples from papillary thyroid carcinoma more than samples from adjacent non-neoplastic thyroid tissue of the same patients ( $\mathrm{p}=0.005$ ). $A P-1$ expression in papillary thyroid cancer tissues is positively associated with advanced stage, lymph nodes metastases $(\mathrm{p}=0.002)$, huge tumor size (0.034), presence of extra-thyroid invasion $(\mathrm{p}=0.005)$, multifocality of the tumor $(\mathrm{p}=0.003)$, vascular invasion $(p=0.02)$, capsular invasion $(p=0.022)$ and distant metastases $(\mathrm{p}=0.042)$ (Table 1 and Figure 2$)$
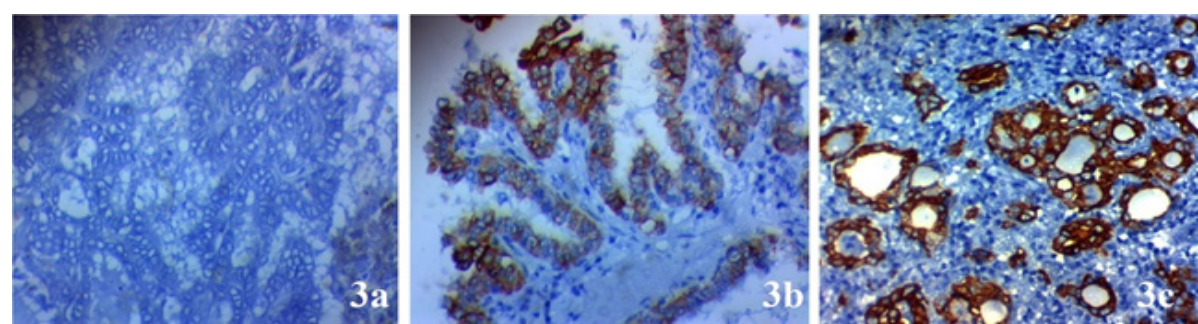

Figure 3. JAZF1 Expression in Papillary Thyroid Carcinoma (PTC). (A) negative cytoplasmic expression in poorly differentiated PTC of high stage $\times 400$, (B) low cytoplasmic expression in well differentiated PTC of low stage $\times 400$, (C) high cy
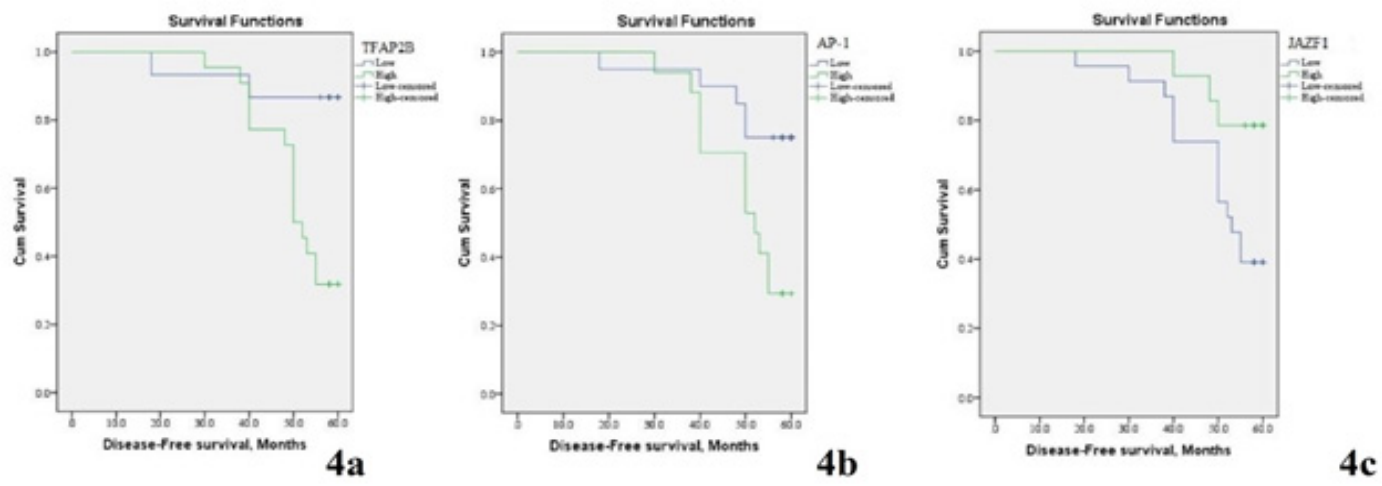

Figure 4. Kaplan Meir Survival Curves of Five-Year Disease Free Survival (DFS) of Included Papillary Thyroid Carcinoma (PTC) Patients. (A) DFS rate stratified according to TFAP2B expression (B) DFS rate stratified according to AP-1 expression, (C) PFS 

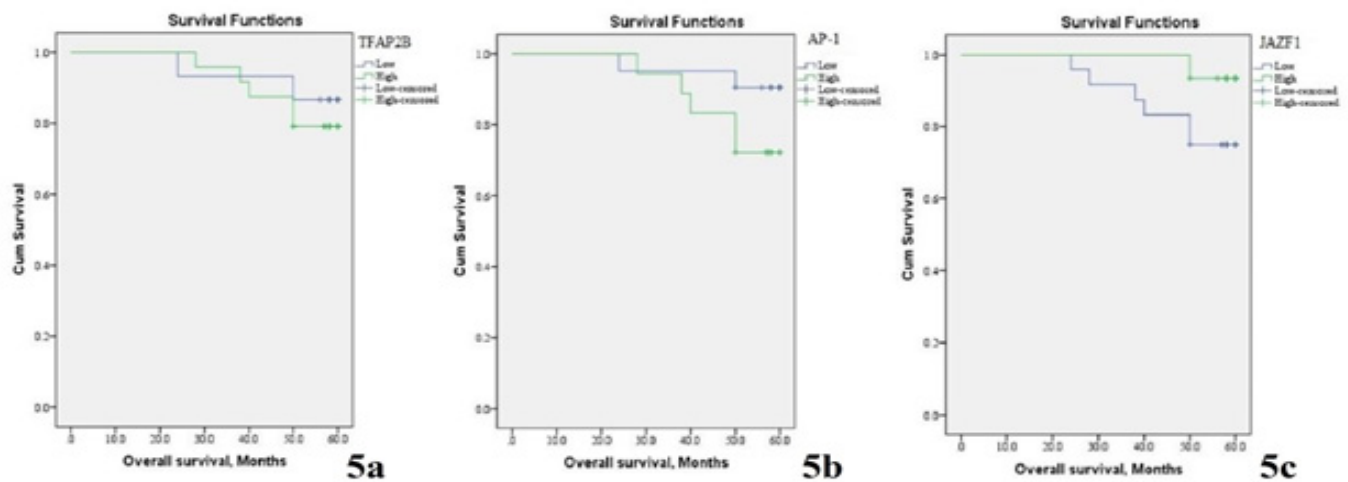

Figure 5. Kaplan Meir Survival Curves of Five-Year Overall Survival Rate (OS) of Included Papillary Thyroid Carcinoma (PTC) Patients. (A) OS rate stratified according to TFAP2B expression (B) OS rate stratified according to AP-1 expression, (C) OS rat

Table 4. Univariate Cox Regression Analyses of Different Prognostic Factors for Overall Survival

\begin{tabular}{lccc}
\hline & HR & $95.0 \%$ CI & P \\
\hline Age $<40$ vs $>40 Y$ & 2.37 & $0.46-12.19$ & 0.303 \\
Sex M vs F & 0.55 & $0.12-2.44$ & 0.427 \\
Histopathological subtype & 1.35 & $0.16-11.21$ & 0.782 \\
TFAP2B, low vs. high & 1.59 & $0.31-8.2$ & 0.58 \\
$A P-1$, low vs. high & 3.10 & $0.6-16.01$ & 0.176 \\
$J A Z F 1$, low vs. high & 0.24 & $0.03-1.97$ & 0.183 \\
Multifocality & 2.08 & $0.46-9.29$ & 0.339 \\
LN involvement & 0.95 & $0.21-4.25$ & 0.947 \\
Vascular invasion & 1.36 & $0.26-7.01$ & 0.714 \\
Capsular invasion & 0.70 & $0.14-3.59$ & 0.665 \\
Extra-thyroid extension & 0.79 & $0.15-4.05$ & 0.773 \\
Stage & 1.45 & $0.66-3.17$ & 0.357 \\
Distant metastasis & 1.92 & $0.37-9.9$ & 0.435 \\
Surgery & 1.45 & $0.68-3.09$ & 0.332 \\
Tumor size & 0.02 & $0.001-12.5$ & 0.239 \\
\hline
\end{tabular}

HR, Hazard ratio; CI, confidence interval

\section{Patients'outcome and survival results}

$A P-1$ expression in papillary thyroid cancer tissues is positively associated with a high incidence of disease progression, recurrence of the tumor $(p=0.005)$, unfavourable disease-free survival rate $(p=0.01)$ (Tables 2-4 and Figures 4-5).

\section{JAZF1 expression in thyroid tissue Immunohistochemistry results}

Expression of $J A Z F 1$ expression was decreased in samples from papillary thyroid carcinoma more than samples from adjacent non-neoplastic thyroid tissue of the same patients $(\mathrm{p}=0.004) . J A Z F 1$ expression in papillary thyroid cancer tissues is negatively associated with advanced stage $(\mathrm{p}=0.34)$, lymph nodes metastases $(p=0.005)$, huge tumor size $(0.023)$, presence of extrathyroid invasion $(p=0.045)$, multifocality of the tumor $(p=0.036)$, vascular invasion $(p=0.007)$ and capsular invasion $(\mathrm{p}=0.037)$ (Table 1 and Figure 3$)$

\section{Patients'outcome and survival results}

$J A Z F 1$ expression in papillary thyroid cancer tissues is negatively associated with a high incidence of disease progression, recurrence of the tumor $(p=0.031)$, unfavourable disease-free survival rate and unfavourable overall survival rate $(\mathrm{p}=0.030)$ (Tables $2-4$ and Figures 4-5). There was a positive association between TFAP $2 B$ and $A P-1$ expression $(\mathrm{r}=+0.460, \mathrm{p}=0.004)$, inverse association between TFAP $2 B$ and $J A Z F 1$ expression $(\mathrm{r}=-0.263, \mathrm{p}=0.003)$ and inverse association between $A P-1$ and $J A Z F 1$ expression $(\mathrm{r}=-0.645, \mathrm{p}=0.005)$.

\section{Discussion}

It is essential to detect novel valid therapeutic target for papillary thyroid cancer mainly for cases resistant to currently used therapies. In our study, we found that TFAP $2 B$ expression was elevated in cells of papillary thyroid cancer in comparison to adjacent non-neoplastic thyroid tissues which are similar to results of $\mathrm{Fu}$ et al., (2019), in papillary thyroid cancer and Fu et al., (2014) in lung cancer. Fu et al., (2019) showed that TFAP2B expression in lung cancer tumor tissue samples is markedly elevated in comparison to healthy tissues.

Moreover, Fu et al., (2019), stated that knocking down TFAP2B in cell lines of thyroid cancer reduced viability, proliferation, migration, and invasion of cancer cells. In our study, we clarified that TFAP $2 B$ higher expression increased the growth of papillary thyroid cancer. That TFAP2B explained these results binds to the promoter of COX-2 in malignant thyroid cells that accelerated cell proliferation and spread so that overexpression of TFAP2B might be rescued by celecoxib which is a COX-2 inhibitor and COX-2 knockdown (Fu et al., 2019).

Additionally, overexpression of TFAP2B stimulated VEGF/PEDF signaling pathway, which has a role in cancer progression. TFAP $2 B$ high expression stimulated cancer progression through activation of many signaling pathways as; caspase and ERK/p38 in lung cancer (Fu et al., 2014).

COX-2 inhibitors might be applied in the clinical therapeutic trials against thyroid cancer to decrease its spread and improve patients' prognosis. Fu et al., (2014), assessed TFAP2B roles in cancer lung progression 
and stated that it is incriminated in vascularization, progression, metastasis, and cancer recurrence, which is similar to results of previous studies (Karjalainen et al., 2000, Schulte et al., 2008, Tellez et al., 2003).

Due to relatively few studies regarding the roles of TFAP2B in cancer progression, we assessed the expression of $A P-1$. We showed that there is up-regulation of the expression of $A P-1$ in thyroid cancer cells and its expression is related to huge tumor size, Similarly Xiao et al., (2019) who showed that AP-1 expression in the group of patient with large tumor size is more than patients with smaller tumors. And it is proved that the size of the tumor of papillary thyroid cancer is a good predictor of recurrence of the tumor and dismal outcome (Ito et al., 2012). AP-1 activation stimulated the expression of $V E G F$ to the increased proliferation of tumor cells (Daft et al., 2015). Additionally, AP-1 increased tumor invasion and metastasis of through regulating matrix metalloproteinase 9 (MMP-9) through interaction with MMP-9 promoter (Motomura et al., 2018). AP-1 inhibitors might lead to blocking angiogenesis and invasion of cancer cells (Dong et al., 2012).

Moreover, MMP-9 up-regulation degraded the extracellular matrix, which caused malignant invasion and progression (Luo et al., 2017). AP-1 increased MMP9 activity that increased esophageal carcinoma cells invasion and metastasis (Shin et al., 2016). We showed that AP-1 was positively associated with the presence of metastasis to the lymph nodes in papillary thyroid carcinoma patients, which is slightly different from Xiao et al., (2019) who showed no statistically significant differences between the expression of $A P-1$ and presence of lymph node spread but they had not clarified the reason.

Different from our findings, Chen et al., (2016) showed a negative association between $A P-1$ expression and tumor size. These different results may be attributed to a different number of patient's different primary antibody used of different methods of assessment. We found a positive association between $T F A P 2 B$ and $A P-1$ expression in papillary thyroid carcinoma patients, but due to a few studies which assessed their expression, we assessed the expression of another biomarker which is JAZF1.

JAZF1 was found to control gluconeogenesis, so, previous studies focused mainly on its role in diabetes and metabolism of lipid (Wei et al., 2018, Jang et al., 2014). Recently, JAZF1 was found to control the biological behaviours of many tumors as endometrial sarcomas and cancer prostate, and it was considered a tumor suppressor (Hodge et al., 2016, Luo et al., 2017, Hazelett et al., 2014). Tumor suppressors have participated in many biological cellular activities as cell proliferation, apoptosis and control of cell cycle (Pospiechet al., 2018, Qiu et al., 2018, Nikbakht et al., 2018, Bai et al., 2018).

We showed that JAZF1 was down-regulated in papillary thyroid cancer tissues more than adjacent nonneoplastic tissues, which was similar to results of Huang et al., (2019), whose report was the first in exploring tumor suppressor role of JAZF1 in papillary thyroid carcinoma using immunohistochemistry and they stated that expression of $J A Z F 1$ in papillary thyroid carcinoma was decreased in comparison with adjacent thyroid tissues or with nodular goiter.

Huang et al., (2019), showed that JAZF1 expression is inversely associated with Ki67 labelling index in cancer cells denoting that JAZF1 made its tumor suppressor function by inhibition of cell proliferation and cell cycle arrest. Yuasa et al., (2015) found different results, showed that increased $J A Z F 1$ expression increased cell proliferation and leads to the progression of the cell cycle, which stimulated oncogenesis in muscle. That JAZF1 role explained this variability differs according to the type of tissue. But the exact role of JAZF1 in cancer is still uncertain needing further studies. Up to our knowledge our study is the first to assess the expression of TFAP $2 B, A P-1$ and $\mathrm{J} A Z F 1$ in malignant and non-neoplastic thyroid tissue using immunohistochemistry and evaluation of novel three markers is the strengths of the study. Further studies are needed to focus on the combined effects of these markers using other methods of assessment as gene expression, which performed on a large number of patients.

In conclusion, we demonstrated that expression levels of TFAP2B and AP-1 protein were increased while the expression levels of $J A Z F 1$ were decreased in papillary thyroid carcinoma in comparison with non-neoplastic thyroid tissues using immunohistochemistry. High expression levels of TFAP $2 B$ and $A P-1$ and low expression levels of $J A Z F 1$ were associated with unfavourable pathological, prognostic parameters and dismal patient's outcome. So the evaluation of tissue protein expression of those three markers might be helpful in the assessment of prognosis of papillary thyroid carcinoma patients.

\section{Acknowledgements}

\section{Author contribution}

AAH-conception and design of the study, acquisition, analysis and interpretation of data, drafting and revising the article and final approval of the version to be published, $\mathrm{OAH}$ and LMG-acquisition and analysis of data, drafting the article and final approval of the version to be published.

\section{Availability of material}

The data of the present study are available with the corresponding author.

\section{Ethics approval and consent to participate Not applicable.}

\section{Consent for publication}

Not applicable.

\section{Conflict of interest}

The authors declare no conflict of interest.

\section{References}

Bai YH, Zhan YB, Yu B, et al (2018). A novel tumor-suppressor, $\mathrm{CDH} 18$, inhibits glioma cell invasiveness via UQCRC2 and correlates with the prognosis of glioma patients. Cell Physiol Biochem, 48, 1755-70.

Chen X, Wu W, Chen X, et al (2016). Roles of phosphatidylinositol 3-kinase regulatory subunit alpha, activator protein-1, and 
programmed cell death 4 in diagnosis of papillary thyroid carcinoma. Tumor Biol, 37, 6519-26.

Daft PG, Yang Y, Napierala D, et al (2015). The growth and aggressive behavior of human osteosarcoma is regulated by a CaMKII-controlled autocrine VEGF signaling mechanism. PLoS One, 10, e0121568.

Dong W, Li Y, Gao M, et al (2012). IKK $\alpha$ contributes to UVB-induced VEGF expression by regulating AP-1 transactivation. Nucleic Acids Res, 40, 2940-55.

Fu H, Zhang H, Chen Z, et al (2019). TFAP2B overexpression contributes to tumor growth and progression of thyroid cancer through the COX-2 signaling pathway. Cell Death Dis, 10, 397.

Fu L, Shi K, Wang J, et al (2014). TFAP2B overexpression contributes to tumor growth and a poor prognosis of human lung adenocarcinoma through modulation of ERK and VEGF/PEDF signaling. Mol Cancer, 13, 89.

Hazelett DJ, Rhie SK, Gaddis M, et al (2014). Comprehensive functional annotation of 77 prostate cancer risk loci. PLoS Genet, 10, e1004102.

Hodge JC, Bedroske PP, Pearce KE, et al (2016). Molecular cytogenetic analysis of JAZF1, PHF1, and YWHAE in endometrial stromal tumors: discovery of genetic complexity by fluorescence in situ hybridization. J Mol Diagn, 18, 516-26.

Huang L, Cai Y, Luo Y, et al (2019). JAZF1 suppresses papillary thyroid carcinoma cell proliferation and facilitates apoptosis via regulating TAK1/NF- $\mathrm{BB}$ pathways. Onco Targets Therapy, 12, 10501-14

Ito Y, Kudo T, Kobayashi K, et al (2012). Prognostic factors for recurrence of papillary thyroid carcinoma in the lymph nodes, lung, and bone: analysis of 5,768 patients with average 10-year follow-up. World J Surg, 36, 1274-8.

Jang WY, Bae KB, Kim SH, et al (2014). Overexpression of Jazf1 reduces body weight gain and regulates lipid metabolism in high fat diet. Biochem Biophys Res Commun, 444, 296-301.

Karjalainen JM, Kellokoski JK, Mannermaa AJ, et al (2000). Failure in post-transcriptional processing is a possible inactivation mechanism of AP-2alpha in cutaneous melanoma. Br J Cancer, 82, 2015-21.

Li JK, Nie L, Zhao YP, et al (2016). IL-17 mediates inflammatory reactions via $\mathrm{p} 38 / \mathrm{c}-\mathrm{Fos}$ and $\mathrm{JNK} / \mathrm{c}-\mathrm{Jun}$ activation in an AP-1-dependent manner in human nucleus pulposus cells. $J$ Transl Med, 14, 77-83.

Luo D, Chen H, Li X, et al (2017). Activation of the ROCK1/ MMP-9 pathway is associated with the invasion and poor prognosis in papillary thyroid carcinoma. Int J Oncol, 51, 1209-18.

Luo Z, Rhie SK, Lay FD, et al (2017). A prostate cancer risk element functions as a repressive loop that regulates HOXA13. Cell Rep, 21, 1411-7.

Motomura H, Seki S, Shiozawa S, et al (2018). A selective c-Fos/AP-1 inhibitor prevents cartilage destruction and subsequent osteophyte formation. Biochem Biophys Res Commun, 497, 756-61.

Nakajima T, Fujino S, Nakanishi G, et al (2004). TIP27: a novel repressor of the nuclear orphan receptor TAK1/TR4. Nucleic Acids Res, 32, 4194-4204.

Nikbakht DM, Azarnezhad A, Hashemibeni B, et al (2018). An effective concentration of 5-Aza-CdR to induce cell death and apoptosis in human pancreatic cancer cell line through reactivating RASSF1A and up-regulation of bax genes. Iran J Med Sci, 43, 533-40.

Pospiech K, Płuciennik E, Bednarek AK(2018). WWOX tumor suppressor gene in breast cancer, a historical perspective and future directions. Front Oncol, 8, 345.
Qiu J, Li X, He Y, et al (2018). Distinct subgroup of the Ras family member 3 (DIRAS3) expression impairs metastasis and induces autophagy of gastric cancer cells in mice. J Cancer Res Clin Oncol, 144, 1869-86.

Schulte JH, Kirfel J, Lim S, et al (2008). Transcription factor AP2alpha (TFAP2a) regulates differentiation and proliferation of neuroblastoma cells. Cancer Lett, 271, 56-63.

Shin WS, Hong Y, Lee HW, et al (2016). Catalytically defective receptor protein tyrosine kinase PTK7 enhances invasive phenotype by inducing MMP-9 through activation of AP-1 and NF- $\mathrm{\kappa B}$ in esophageal squamous cell carcinoma cells. Oncotarget, 7, 73242-56.

Tellez C, McCarty M, Ruiz M, et al (2003). Loss of activator protein-2alpha results in overexpression of protease-activated receptor-1 and correlates with the malignant phenotype of human melanoma. J Biol Chem, 278, 46632-42.

Tewari D, Nabavi SF, Nabavi SM, et al (2018). Targeting activator protein 1 signaling pathway by bioactive natural agents: possible therapeutic strategy for cancer prevention and intervention. Pharmacol Res, 128, 366-75.

Wei Q, Zhou B, Yang G, et al (2018). JAZF1 ameliorates age and diet-associated hepatic steatosis through SREBP-1c -dependent mechanism. Cell Death Dis, 9, 859.

Xiao Ch, Huang Y, Gao Q, et al (2019). Expression of activator protein-1 in papillary thyroid carcinoma and its clinical significance. World J Surg Oncol, 17, 25-32.

Xu X, Liu Z, Huang H, et al (2017). AP-2alpha and AP-2beta regulate dorsal interneuron specification in the spinal cord. Neuroscience, 340, 232-42.

Yuasa K, Aoki N, Hijikata T (2015). JAZF1 promotes proliferation of $\mathrm{C} 2 \mathrm{C} 12$ cells, but retards their myogenic differentiation through transcriptional repression of MEF2C and MRF4-implications for the role of Jazf1 variants in oncogenesis and type 2 diabetes. Exp Cell Res, 336, 287-97.

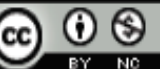

This work is licensed under a Creative Commons AttributionNon Commercial 4.0 International License. 